\title{
Colaboración intradisciplinar en Trabajo Social: explorando el peso explicativo de aspectos institucionales, organizacionales y personales en la colaboración entre profesionales y técnicos en Chile
}

Intradisciplinary collaboration in Social Work: exploring the weight of institutional, organizational, and personal aspects of cooperation between professionals and technicians in Chile.

Colaboração intradisciplinar em Trabalho Social: explorando o peso explicativo de aspectos institucionais, organizacionais e pessoais na colaboração entre profissionais e técnicos no Chile

Carlos Andrade Guzmán*

\section{RESUMEN}

Reconociendo que la colaboración en el contexto de la intervención es, primero, relevante, y, segundo, que ha sido principalmente estudiada desde una perspectiva interdisciplinar, este estudio, situado en el Trabajo Social chileno, observa la colaboración intradisciplinar, específicamente entre profesionales y técnicos/as en Trabajo Social. Busca responder, entre otras preguntas, en qué medida la institucionalidad, los aspectos organizacionales y los elementos personales de los interventores incidirían en la colaboración intradisciplinar. Metodológicamente, con base en un cuestionario cuantitativo, respondido por profesionales y técnicos/as en Trabajo Social en Chile y utilizando un modelo de regresión lineal múltiple, este trabajo responde las preguntas que lo han guiado. En términos de resultados, entre otros, se advierte que estos elementos explicarían cuantitativamente, de forma importante, la colaboración. Entre sus conclusiones, se advierte la necesidad de fortalecer las condiciones ins-
Palabras clave: Trabajo Social, Chile, colaboración intradisciplinar, profesionales y técnicos del Trabajo Social

* Chileno. Doctor (c) en Trabajo Social, Universidad Alberto Hurtado, Chile y PhD (c) in Social Welfare, Boston College, Estados Unidos. Académico e investigador del Departamento de Trabajo Social de la Facultad de Ciencias Sociales de la Universidad Alberto Hurtado. caandrade@uahurtado.cl 
titucionales y organizacionales para favorecer una colaboración significativa en pos de la intervención.

\section{SUMMARY}

Recognizing that collaboration in the context of intervention is, first, relevant, and second, that it has been mainly studied from an interdisciplinary perspective, this study, situated in Chilean Social Work, looks at intradisciplinary collaboration, specifically between professionals and technicians in Social Work. It seeks to answer, among other questions, to what extent the interveners' institutional, organizational, and personal aspects would influence intradisciplinary collaboration. Methodologically, based on a quantitative questionnaire, answered by professionals and technicians in Social Work in Chile and using a multiple linear regression model, this work answers the questions that have guided it. In terms of results, it is noted that these elements would undoubtedly explain the collaboration quantitatively. Among its conclusions, the need to strengthen institutional and organizational conditions to favor meaningful cooperation in pursuit of intervention is noted.

\section{RESUMO}

Reconhecendo que a colaboração no contexto da intervenção é, primeiro, relevante e que, segundo, tem sido estudada principalmente sob uma perspectiva interdisciplinar, este estudo, situado no Trabalho Social chileno, observa a colaboração intradisciplinar, especificamente entre profissionais e técnicos(as) em Trabalho Social. Busca responder, entre outras perguntas, em que medida a institucionalidade, os aspectos organizacionais e os Palavras-chave: Trabalho Social, Chile, colaboração intradisciplinar, profissionais e técnicos do Trabalho Social elementos pessoais dos interventores incidiriam na colaboração intradisciplinar. Metodologicamente, com base em um questionário quantitativo, respondido por profissionais e técnicos(as) em Trabalho Social no Chile e utilizando um modelo de regressão linear múltipla, este trabalho responde as perguntas que o guiaram. Em termos de resultados, entre outros, adverte-se que estes elementos explicariam quantitativamente, de forma importante, a colaboração. Entre suas conclusões, adverte-se a necessidade de fortalecer as condições institucionais e organizacionais para favorecer uma colaboração significativa após a intervenção.

Key words: Social Work, Chile, intradisciplinary collaboration, social work, social work professionals and technicians 


\section{Introducción}

Desde que emerge como profesión, en 1925, el Trabajo Social chileno ha focalizado su acción en fenómenos sociales como la pobreza (Vidal, 2015) y la exclusión social (Muñoz-Arce, 2016). En esta línea, para alcanzar sus objetivos de intervención, una característica de su acción ha sido el trabajo colaborativo (González-Celis y Pérez-Roa, 2009; López, 2010), por ejemplo, a través de intervenciones disciplinares o interdisciplinares (o interprofesionales ${ }^{1}$ ). En relación a estas experiencias colaborativas, el trabajo interdisciplinar y sus características y desafíos ha sido estudiado en Chile en relación con sus implicancias para los sujetos de la intervención (González-Celis y Pérez-Roa, 2009; González, 2009; Muñoz-Arce, 2014; Muñoz-Arce y Madrigal-Calderón, 2018). Sin embargo, desde una perspectiva general, poca atención se ha puesto en el análisis de la colaboración entre profesionales de la disciplina del Trabajo Social en Chile. Menos aún en relación con la colaboración entre profesionales y técnicos del área. Contribuir a disminuir esta brecha en el campo de conocimiento intradisciplinar es el propósito de este estudio.

Avanzar en la comprensión de aspectos vinculados a la colaboración entre personas interventoras del Trabajo Social en Chile es relevante, debido a que diversa investigación nacional e internacional ha reconocido la necesidad de la colaboración a la hora de abordar fenómenos sociales complejos (Jacob, Boshoff, Stanley, Stewart, \& Wiles, 2017; Katherine \& Georgeson, 2013; Morgan, Pullon, \& Mckinlay, 2015; Muñoz-Arce, 2014, 2016; Muñoz-Arce y Madrigal-Calderón, 2018; Orchard, 2010; Ross, 2009; Smith, 2015; West, Miller \& Leitch, 2016). No obstante, esta investigación ha tendido a poner atención en la colaboración basada, principalmente, en el enfoque de la interdisciplina o interprofesionalidad (véanse, entre otras, las contribuciones de Hannah \& Jindal-Snape (2014) o los aportes de MuñozArce y Madrigal-Calderón (2018), esto es, en el encuentro para llevar adelante estrategias colaborativas entre actores de diversas áreas de

1 Reconociendo las diferencias entre los conceptos de "disciplina" y "profesión", en adelante se utilizarán indistintamente los conceptos de interdisciplina o interprofesionalidad para referir a la articulación entre actores de diferentes áreas de formación. 
formación, como, por ejemplo, combinaciones entre la psicología, la enfermería y el trabajo social.

Dentro de este contexto, avanzar en la comprensión del encuentro colaborativo entre interventores del Trabajo Social es relevante en materia de estudios disciplinares, debido a que el Trabajo Social chileno es altamente heterogéneo (Muñoz-Arce, 2018), encontrándose fuertemente fragmentado, lo cual se muestra en que actualmente

existen 107 ofertas de programas universitarios de Trabajo Social; 97 de Servicio Social ofrecidos por Institutos Profesionales; 17 programas de Trabajo Social en Institutos Profesionales; 61 técnicos en Trabajo Social dictados por Centros de Formación Técnica; 50 programas de técnicos en Servicio Social y 94 de técnicos en Trabajo Social dictados por Institutos Profesionales, lo que da un total de 426 ofertas de formación terciaria. (Iturrieta, 2017, p. 17)

Este elemento, configura al Trabajo Social chileno como un campo altamente diverso, con interventores con diferente tipo de formación en relación a aspectos teóricos, epistémicos y metodológicos de intervenir, por señalar solo algunos elementos que permearían, de alguna forma, las estrategias colaborativas que desarrollan en el marco de la implementación de programas sociales.

En este sentido, la heterogeneidad propia del Trabajo Social en general (Sharland, 2012), así como de la disciplina en Chile en particular (Iturrieta, 2017), cristaliza lo lejano a la realidad de considerar al Trabajo Social en el país como a un campo único e indiferenciado, volviendo relevante la necesidad de profundizar en la comprensión de aspectos vinculados con la colaboración en marcos de encuentro intradisciplinar.

Cabe relevar que diversa investigación nacional e internacional ha puesto de relieve cómo diversos aspectos permearían la forma de colaborar en contextos de intervención social. Al respecto, la colaboración podría estar influenciada por aspectos institucionales y organizacionales dados por el lugar desde el cual los interventores desarrollan su actuación disciplinar (Andrade-Guzmán, Martin y Martínez, 2014; Barrow, Barrow \& Glockling, 2014; Calkin, 1988; Caplan \& Ricciardelli, 2016; Hodson \& Deery, 2014; Kelly \& Young, 2014), como, por 
ejemplo, la institucionalidad que orienta los programas sociales. Asimismo, por las condiciones que se generan para ello, por ejemplo, el número de casos con el que se debe intervenir. Por su parte, las estrategias colaborativas podrían estar permeadas por diversos elementos organizacionales (Galyean, Lawson, Jones, Dreyfus \& Berrick, 2018): aspectos de cultura organizacional, la cantidad de trabajo administrativo que deben gestionar los interventores, las características del estilo directivo de las jefaturas directas, el contar con tiempo y espacios para el autocuidado o la reflexión sobre los procesos interventivos, por señalar algunos casos.

Por su parte, diversa investigación nacional e internacional ha puesto de relieve cómo la colaboración podría estar influenciada por aspectos personales, lo cual sería extensible a la colaboración intradisciplinar en Trabajo Social. Ello debido a que los interventores llevarían consigo diversas trayectorias basadas en, entre otros elementos, experiencias familiares, vocacionales y políticas, por señalar algunos que posiblemente explicarían la forma en que entienden y ejercen su práctica (Aguayo-Cuevas, Cornejo-Torres y López-Vásquez, 2018) cuando deben trabajar con otros y otras en contextos de intervención. En esta línea, los interventores serían el resultado de sus propias historias y de la interacción con otras personas, encuentro en el que estarían envueltos, entre otros, motivaciones y la propia agencia (Holland \& Lave, 2009), así como también sus sistemas de creencias (Hodson \& Deery, 2014), los valores personales y morales, las influencias propias de la cultura y otros elementos como la religión, el género, la etnicidad y los aspectos familiares y comunitarios (Hannah \& Jindal-Snape, 2014), que podrían incidir en la forma de trabajar con otras y otros en contextos de intervención.

Cabe relevar, sin embargo, que, en términos generales, esta investigación nuevamente ha tendido a aproximarse desde un enfoque interprofesional o interdisciplinar, aumentando la relevancia de profundizar en el conocimiento respecto de la colaboración desde una perspectiva intradisciplinar centrada en el Trabajo Social chileno.

Estos elementos han conformado el problema de investigación que aborda este trabajo. Así, tomando como referencia los elementos institucionales, organizacionales y personales relevados por diversa investigación nacional e internacional, como aspectos que incidirían en la 
colaboración en contextos de intervención, este trabajo busca, desde una perspectiva intradisciplinar y apoyada en un diseño cuantitativo, responder a las siguientes preguntas de investigación: 1) ¿en qué medida aspectos institucionales, organizacionales y personales explicarían la colaboración desarrollada por profesionales y técnicos del Trabajo Social en Chile? y, 2) ¿cuál de estos elementos, tendría un mayor peso explicativo en la colaboración llevada adelante por interventores de la disciplina?

Cabe señalar que, dada la importancia que la colaboración tiene para el abordaje de fenómenos sociales complejos en contextos de intervención, este estudio se ha justificado, primeramente, desde un criterio de relevancia social. En segundo lugar, desde un criterio de valor teórico, en términos de poder contribuir a densificar el cuerpo de literatura que, centrado específicamente en el caso chileno, reflexiona sobre elementos de la colaboración intradisciplinar desde el Trabajo Social.

\section{Posicionamiento paradigmático}

La posición paradigmática en este estudio es crítica (Guba \& Lincoln, 2005; Pérez, 1994). En este sentido, este trabajo entiende que los interventores del Trabajo Social que implementan programas sociales enfrentan tradicionalmente fenómenos altamente complejos y, por tanto, contar con las mejores condiciones para poder colaborar con otras y otros en el marco de su actuación disciplinar supone un imperativo ético a la hora de dar respuesta a las necesidades que enfrentan los sujetos de intervención. En este sentido, aun cuando este estudio ha basado su producción de datos en estrategias cuantitativas de investigación, en todos sus componentes se sostiene en la posición crítica de su investigador basada en su propia trayectoria, tanto en la intervención como en la investigación, en un ejercicio permanente de reflexión sobre cómo proveer de mejores condiciones a los equipos para el desarrollo de políticas e intervenciones sociales, desde un reconocimiento de la importancia que reviste para los sujetos beneficiarios, así como de la relevancia que tiene dignificar, en todos los casos, las condiciones con las que los interventores cuentan para llevar adelante su actuación. 


\section{Perspectiva teórica: el institucionalismo crítico}

La presente investigación se basa en el institucionalismo crítico (Cleaver, 2002; Cleaver \& De Koning, 2015; Cleaver \& Whaley, 2018) para abordar la reflexión sobre colaboración intradisciplinar entre personas profesionales y técnicas del Trabajo Social, en el contexto de programas sociales en Chile.

El institucionalismo crítico pone el acento en la complejidad de las instituciones que se intersectan en la vida social, su proceso histórico de formación, las interacciones entre instituciones de orden formal e informal, y las relaciones de poder que se encuentran envueltas en dichas instituciones (Cleaver \& De Koning, 2015). En este sentido, reconoce que la complejidad es una característica propia de los sistemas sociales y de la interfaz entre las personas y el ambiente, así como también el que esta se manifiesta en identidades diversas, en una pluralidad institucional y en la intersección de campos locales y globales (Cleaver \& Whaley, 2018). Para esta perspectiva, las instituciones serían el conjunto de normas, reglas y prácticas que necesitarían de la estructura social, de la historia, de los significados, de las relaciones de poder y de la legitimidad para funcionar (Cleaver \& Whaley, 2018). Desde una comprensión del cambio institucional que sigue la idea de Levi-Strauss, el institucionalismo crítico entiende que la modificación de las instituciones se produce sobre la base de un ejercicio de bricolaje institucional (Cleaver \& De Koning, 2015), que correspondería a un proceso por medio del cual las personas, de forma consciente o no, ensamblan y remodelan los arreglos institucionales, recurriendo a aquello de lo que disponen para hacerlo, independientemente del propósito original para el que fueron creados dichos elementos. En este sentido, "las instituciones así formadas son, por tanto, un mosaico (patchwork) de lo nuevo y de segunda mano, e incluyen: formas habituales de hacer las cosas; prácticas desgastadas adaptadas a las nuevas condiciones; arreglos organizacionales creados o tomados prestados de otros lugares" (Cleaver \& De Koning, 2015, p. 5), y que orientarían el hacer de los actores sociales. En esta línea, los aspectos organizacionales cobrarían relevancia en función de que, desde una perspectiva estructural, estos, sumados a los aspectos institucionales, tendrían influencia en el actuar de las personas.

Así, la idea de "agencia" sería central. Esta referiría a las diferentes formas de comportamiento, conscientes y no conscientes, a tra- 
vés de las cuales las personas usan sus capacidades para actuar en diálogo con sus identidades sociales (Cleaver \& Whaley, 2018). Este ejercicio de agencia sería formado por dinámicas de poder, incluido aquel implícito en la asignación de recursos y en los arreglos institucionales de la sociedad (Cleaver \& De Koning, 2015). En una perspectiva dialéctica entre agencia y estructura, esta propuesta se ocupa de observar cómo la primera es facilitada o restringida por la segunda, mientras, en paralelo, cómo la propia agencia reproduce y transforma dicha estructura y los recursos derivados para la acción (Cleaver \& Whaley, 2018).

En este contexto, la propuesta del institucionalismo crítico contribuye a enriquecer la aproximación hacia la colaboración en Trabajo Social. Entre otros, su abordaje de agencia provee un marco comprensivo para aproximarse a las estrategias colaborativas en sí. Por su parte, el reconocimiento de que los ejercicios de agencia son formados por dinámicas de poder, arreglos institucionales y organizacionales, así como también de aspectos personales, permite contar con un marco para abordar los elementos que estarían a la base de la colaboración. Finalmente, su propio reconocimiento de la complejidad de las instituciones posibilita contar con un marco para comprender las dinámicas inherentemente complejas que se presentan en el desarrollo de intervenciones sociales.

\section{Metodología}

\section{Diseño, alcance del estudio y muestra}

La investigación se inscribió en un estudio financiado por el Fondo de Fomento a la Investigación, de la Vicerrectoría de Investigación y Postgrado de la Universidad Alberto Hurtado. El estudio, basado en un diseño mixto, ha seguido la estructura cualitativa-cuantitativa-cualitativa. Para efectos de este artículo, se presentan resultados basados en su vertiente cuantitativa. Cabe relevar que, metodológicamente, la investigación se inscribe en un estudio de caso colectivo (Stake, 1999) de carácter instrumental, centrado en dos ámbitos de la intervención social: a) infancia y b) discapacidad. La selección de estos casos se basó en los siguientes criterios: 1) en ambos, los equipos de intervención tienden a estar conformados por, entre otros, trabajadores (o asisten- 
tes) sociales y técnicos en Trabajo Social (o Servicio Social); 2) en los casos en los que los equipos de intervención no están conformados por personas técnicas y profesionales, en estos ámbitos tiende a existir la necesidad de establecer estrategias de colaboración con interventores profesionales y técnicos en Trabajo Social de otras organizaciones, 3) los casos, al dirigirse a distintos sujetos de intervención, posibilitan tener un acercamiento mayormente comprehensivo a los aspectos de colaboración, principalmente desde un reconocimiento de la alta complejidad que tiende a enfrentarse en las intervenciones en infancia y discapacidad. Su carácter instrumental se basó en que posibilitó aproximar claves para la reflexión sobre la colaboración intradisciplinar en general, a partir de las experiencias de los interventores en estos ámbitos.

Dentro de este marco, la vertiente cuantitativa que se presenta en este trabajo ha tenido un alcance exploratorio-explicativo. Exploratorio, dado que la observación de aspectos de colaboración intradisciplinar entre profesionales y técnicos del Trabajo Social en Chile corresponde a un espacio, en términos relativos, poco estudiado. Explicativo, en tanto busca aproximar cómo los aspectos institucionales, organizacionales y personales explicarían la colaboración en la intervención.

En términos muestrales, el diseño fue no probabilístico, de tipo intencionado, por criterios (Marradi, Archenti y Piavoni, 2010) y por conveniencia (Cea, 2001), convocando a participar voluntariamente a personas trabajadoras sociales y técnicas chilenas que cumplieran con los siguientes criterios de inclusión: 1) poseer un título profesional o técnico en el área de Trabajo Social (o Servicio Social), 2) participar en la implementación de iniciativas en los ámbitos de infancia o de discapacidad, 3) tener la necesidad de desarrollar acciones colaborativas durante la implementación con otros actores del Trabajo Social (por ejemplo, definir conjuntamente estrategias de intervención, compartir información sobre los sujetos, entre otros). La producción de datos, se acompañó, además, de la estrategia de bola de nieve. En este marco, se presenta el análisis basado en la información de, originalmente, 106 interventores del Trabajo Social que participaron en el estudio a lo largo de todo Chile. La única región de la que no se pudo producir información fue la de Tarapacá. Tomando estos elementos en conside- 
ración, a continuación, se presentan los estadísticos descriptivos correspondientes a la muestra:

Tabla 1.

Estadísticos descriptivos de la muestra

\begin{tabular}{|c|c|c|}
\hline Variables & Porcentaje & Media \\
\hline \multicolumn{3}{|l|}{ Género } \\
\hline Femenino & 72,6 & \\
\hline Masculino & 27,4 & \\
\hline \multicolumn{3}{|l|}{ Título } \\
\hline Profesional del Trabajo Social & 84 & \\
\hline Técnica/o del Trabajo Social & 16 & \\
\hline \multicolumn{3}{|l|}{ Régimen de estudios } \\
\hline Diurno & 74,5 & \\
\hline Vespertino & 25,5 & \\
\hline \multicolumn{3}{|l|}{ Región en la cual interviene } \\
\hline Antofagasta & 0,9 & \\
\hline Arica y Parinacota & 0,9 & \\
\hline Atacama & 0,9 & \\
\hline Aysén del G. Carlos Ibáñez del Campo & 0,9 & \\
\hline Biobío & 3,8 & \\
\hline Coquimbo & 0,9 & \\
\hline La Araucanía & 3,8 & \\
\hline Libertador General Bernardo O'Higgins & 0,9 & \\
\hline Los Lagos & 2,8 & \\
\hline Los Ríos & 1,9 & \\
\hline Magallanes y de la Antártica Chilena & 3,8 & \\
\hline Maule & 0,9 & \\
\hline Metropolitana de Santiago & 68,9 & \\
\hline Ñuble & 1,9 & \\
\hline Valparaíso & 6,6 & \\
\hline \multicolumn{3}{|l|}{ Área de intervención } \\
\hline Discapacidad & 17,9 & \\
\hline Infancia & 82,1 & \\
\hline \multicolumn{3}{|l|}{ Tipo de organización en que trabaja } \\
\hline Privada & 4,7 & \\
\hline Sociedad civil & 52,8 & \\
\hline Pública & 42,5 & \\
\hline Rol que cumple & & \\
\hline
\end{tabular}




\begin{tabular}{lc}
\hline $\begin{array}{l}\text { Coordinación (técnica y/o administrativa- } \\
\text { financiera) }\end{array}$ & 24,5 \\
\hline Coordinación e intervención directa & 37,7 \\
\hline Intervención directa (sin labores de coordinación) & 37,7 \\
\hline Conformación del equipo & 82,1 \\
\hline $\begin{array}{l}\text { Equipo conformado por trabajadores sociales y } \\
\text { técnicos en Trabajo Social }\end{array}$ & 17,9 \\
\hline $\begin{array}{l}\text { Equipo no conformado por trabajadores sociales y } \\
\text { técnicos en Trabajo Social, pero para la intervención } \\
\text { se debe vincular colaborativamente con otros } \\
\text { profesionales y técnicos del Trabajo Social }\end{array}$ & \\
\hline Edad de la persona interventora (años) & 35,2 \\
\hline
\end{tabular}

Fuente: Elaboración propia

\section{Instrumento y análisis de datos}

En el primer momento cualitativo del proyecto en el cual se inscribe este trabajo, se produjo información que permitió, entre otros, identificar diversos elementos a incorporar en el cuestionario cuantitativo aplicado en línea, del cual se presentan resultados en este artículo. Asimismo, a este cuestionario se integraron diferentes elementos identificados sobre la base de revisión de literatura y de la propuesta teórica del institucionalismo crítico. En este contexto, se elaboró una primera versión del cuestionario, la cual fue compartida con un comité asesor conformado por dos personas técnicas en Trabajo Social: una que interviene en el ámbito de infancia y otra en discapacidad. Por su parte, el instrumento fue compartido con dos personas trabajadoras sociales, con experiencia en la colaboración entre profesionales y técnicos de la disciplina en los ámbitos de infancia y discapacidad, y con experiencia docente en la formación de trabajadores y técnicos en Trabajo Social. Con cada una de estas personas se sostuvieron reuniones que permitieron revisar la totalidad de ítems del cuestionario. Este proceso permitió, entre otros aspectos, ajustar su fraseo, evaluar el incorporar o quitar algunos, revisar las categorías de respuesta, así como también aspectos vinculados a la extensión del mismo. Posteriormente, se probó la funcionalidad del instrumento, por medio de una etapa de pretest, la cual permitió hacer los ajustes finales. El cuestionario definitivo fue construido desde una operacionalización que consideró, entre 
otras, una dimensión institucional, una de elementos organizacionales y otra de elementos personales. Asimismo, una de estrategias colaborativas. En el contexto de estas dimensiones, 45 afirmaciones planteadas en el sentido de favorecimiento de la colaboración en el marco de la institucionalidad, los aspectos organizacionales y los elementos personales, y de descripción acciones positivas en función de las diversas estrategias colaborativas en el marco del encuentro intradisciplinar, fueron evaluadas en escalas ordinales de siete categorías, que transitaban desde $1=$ "extremadamente en desacuerdo" a $7=$ "extremadamente de acuerdo". Cabe relevar que los datos en los que se basa este trabajo fueron producidos entre agosto y octubre de 2020. El cuestionario fue difundido en diferentes redes sociales y, asimismo, distribuido de manera personalizada con técnicos y profesionales del Trabajo Social. A quienes respondieron se les pidió difundir el cuestionario con otras personas interventoras de la disciplina que cumplieran los criterios de selección muestral, de manera de aumentar el número de respuestas. Cabe señalar que, en términos éticos, todo el estudio ha contado con la aprobación del Comité de Ética de la Universidad Alberto Hurtado. En este contexto, la primera pregunta del cuestionario en línea correspondió al consentimiento informado, produciéndose información únicamente de quienes consintieron participar.

En términos de la técnica de análisis de los datos presentados en este artículo, dadas las preguntas de investigación que le orientan, se ha optado por el análisis estadístico multivariable, con base en un modelo de regresión lineal múltiple. Debido a que el modelo de regresión lineal requiere metodológicamente que la variable dependiente sea cuantitativa, de tipo continuo, de manera de no violar su supuesto de distribución normal de los residuos y su varianza constante (Cea, 2004), se tomó la decisión metodológica de construir escalas sobre la base de valores medios, tomando como referencia las afirmaciones del cuestionario. Esto permitió que aquellas originalmente de acuerdo/desacuerdo en ordinalidad, pudiesen ser comprehensivamente reunidas en escalas continuas. Asimismo, permitió mantener el rango y sentido interpretativo desde 1 a 7 , indicando que, mientras más cerca estuviese la persona del valor 7 en las respectivas escalas, su percepción estaría más de acuerdo en relación a cómo cada variable 
favorecería la colaboración entre profesionales y técnicos del Trabajo Social en el caso de la institucionalidad, los aspectos organizacionales y los elementos personales, y en relación a la descripción de acciones positivas en el caso de la colaboración. Mientras más cerca del valor 1, en cambio, menos de acuerdo estaría. Cabe mencionar que, de modo de construir escalas metodológicamente plausibles, se estudió la fiabilidad de cada una de ellas en base al análisis de alfa de Cronbach. En todos los casos, las escalas superaron umbrales de 0,850, lo cual se consideró adecuado, en tanto sobrepasaban el umbral teóricamente aceptado de 0,700 . Se presentan a continuación los resultados de los análisis de fiabilidad de las escalas en función de los ítems a la base de las afirmaciones del cuestionario.

Tabla 2.

Análisis de fiabilidad de las escalas

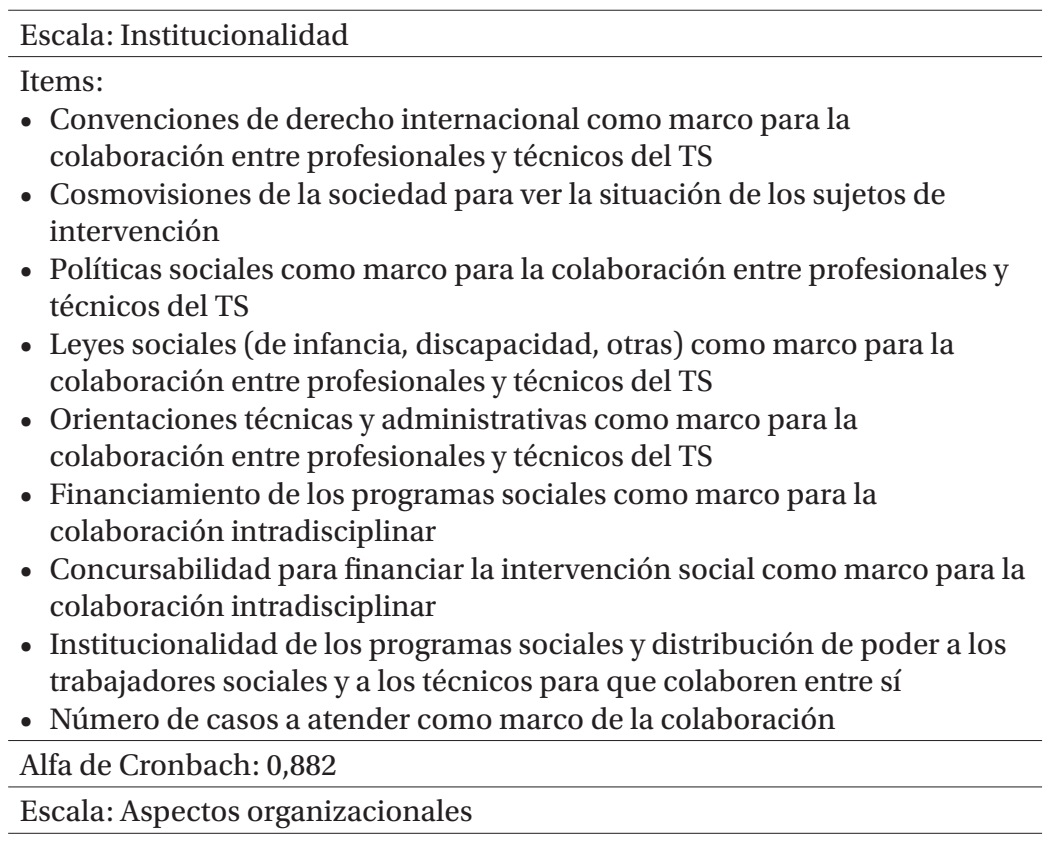


Items:

- Organizaciones/entidades ejecutoras y reconocimiento y valoración de la contribución de trabajadores y técnicos en trabajo social como marco de la colaboración

- Organizaciones y promoción de una relación horizontal con poder compartido entre trabajadores y técnicos como condición de colaboración

- Concentración territorial de los casos a intervenir como condición para la colaboración

- Cantidad de trabajo administrativo como condición para la colaboración

- Recursos materiales para intervenir como condición de la colaboración

- Estilo directivo de las jefaturas directas como condición de la colaboración

- Reuniones de trabajo y otros encuentros en los que se puede compartir con confianza experiencias, inquietudes y problemáticas como condición para la colaboración

- Modelos técnicos de intervención de las organizaciones como condición de la colaboración

- Instrumentos de monitoreo a la gestión como marco de la colaboración

- Espacios para el autocuidado de los equipos como condición para la colaboración

- Sueldo que reciben los miembros de los equipos de intervención como condición de la colaboración

- Contar con contrato de trabajo (no a honorarios) como condición de la colaboración

- Delimitación de funciones y de roles como condición de la colaboración

- Nivel de rotación en los equipos como marco de la colaboración

- Contar con seguro de accidentes como condición para la colaboración

Alfa de Cronbach: 0,867

Escala: Elementos personales

Items:

- Miradas de entender el mundo y sistemas de creencias que tienen los interventores como condición de colaboración

- Reconocimiento y valoración de la contribución del otro como condición de la colaboración entre técnicos y profesionales del trabajo social

- Actitud de los interventores para promover un acercamiento horizontal como condición de la colaboración

- Compromiso y responsabilidad con la intervención como condición para la colaboración

- Empatía como condición de la colaboración

- Asertividad

- Confianza en el trabajo del otro como condición para la colaboración

- Confianza en el trabajo de una/o como condición para la colaboración

- Flexibilidad ante el cambio como condición para la colaboración

- Disposición a ceder poder como condición para la colaboración

- Respeto mutuo como condición de la colaboración

- Saber escuchar al otro como condición de la colaboración 


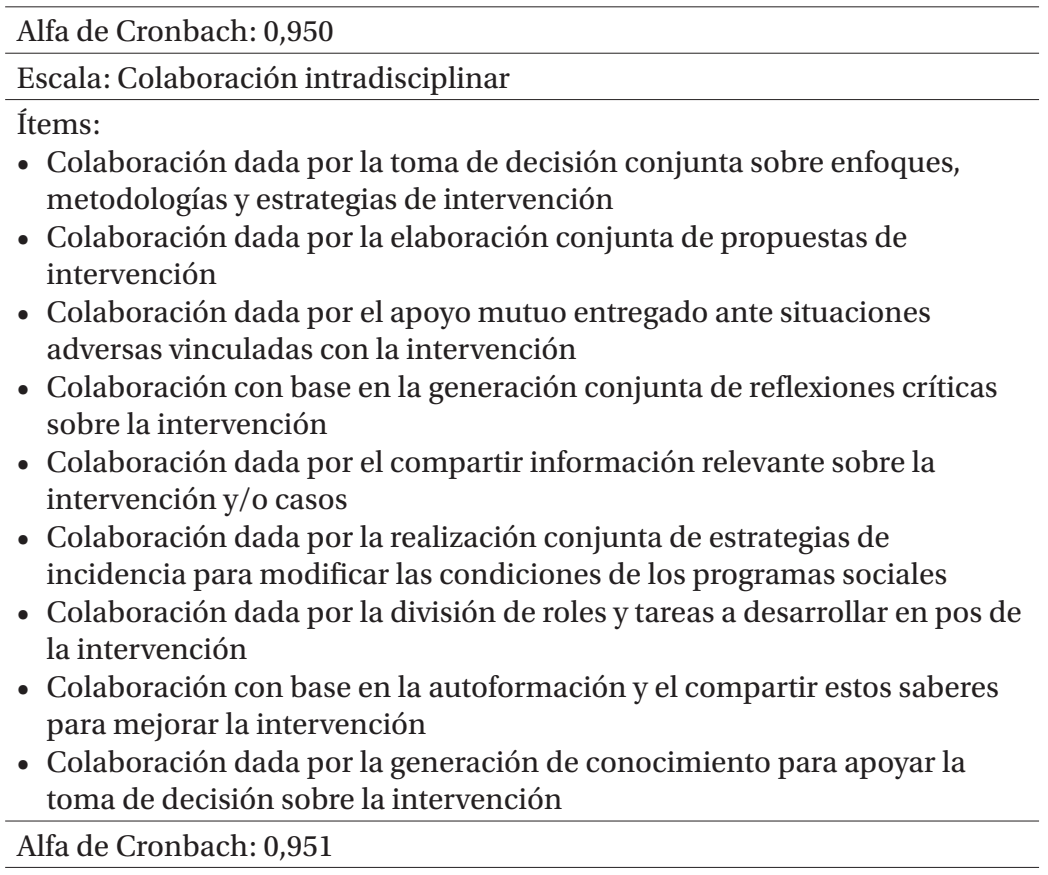

Fuente: Elaboración propia.

Es necesario mencionar que, previo al modelamiento con base en regresión lineal, las cuatro escalas (en adelante, variables o variables comprehensivas) fueron evaluadas en función de si existía o no correlación entre ellas, de manera de posteriormente ser ingresadas al modelo. Esto se llevó a cabo sobre la base de matrices de correlaciones de Pearson. En todos los casos se presentó correlación. Considerando las preguntas de investigación, la colaboración intradisciplinar correspondería a la variable dependiente, mientras que la institucionalidad, los aspectos organizacionales y los elementos personales a las independientes. Para el modelo se consideró el total de casos de personas interventoras sobre las que hubiera información en las cuatro variables a modelar: 104 observaciones. Ello en función de que la diferencia de dos casos respecto a los 106 que contestaron el cuestionario, corresponde a observaciones con valores perdidos en algunas respuestas del cuestionario. Metodológicamente se optó por no imputar respuestas en estos casos. En este sentido, cumpliéndose con la mayoría de los supuestos metodológicos para elaborar un modelo de regresión, en una primera prueba se advirtió que el coeficiente asociado al intercepto (beta cero) era el único del modelo 
que no daba significativo en términos estadísticos. Asimismo, los residuos no distribuyeron de manera normal. En este marco, se identificaron algunos valores atípicos estadísticamente en el conjunto de datos. Se hicieron nuevas pruebas al filtrar un solo caso de los atípicos, lo que permitió arribar a un modelo en que los valores residuales distribuyeran de forma normal y se cumpliera con el conjunto de supuestos primordiales para elaborar metodológicamente un modelamiento con regresiones de este tipo (linealidad entre las variables, normalidad y homocedasticidad de residuos y baja multicolinealidad). El beta asociado al intercepto, no obstante, siguió siendo no significativo. Sin embargo, dado que, para efectos metodológicos e interpretativos, este elemento no es el de mayor relevancia (Gujarati y Porter, 2010), se consideró el modelo como adecuado para los efectos de abordar las preguntas de investigación. Finalmente, cabe relevar que todos los análisis fueron asistidos por SPSS 19. Tomando en consideración estos elementos, se presentan a continuación los resultados de este trabajo.

\section{Resultados}

Un primer hallazgo en sí mismo es que, para los casos de la muestra, las cuatro variables - colaboración intradisciplinar, institucionalidad, aspectos organizacionales y elementos personales- se encuentran correlacionadas en términos estadísticos. Ello presentando altas correlaciones de Pearson de orden positivo, indicando que, por ejemplo, a medida que aumenta el acuerdo con relación a cómo la institucionalidad favorecería la colaboración intradisciplinar, también aumentaría el acuerdo en relación con las expresiones positivas de la colaboración. Situadamente con base en los datos que se disponen, esto ocurriría también para el caso de los elementos personales y de los organizacionales respecto de la colaboración entre interventores del Trabajo Social. Se presentan los resultados del análisis de correlación en la siguiente tabla:

Tabla 3.

Correlación de Pearson entre "colaboración intradisciplinar," "institucionalidad", "aspectos organizacionales" y "elementos personales"

\begin{tabular}{lccc}
\hline Correlación de pearson & Institucionalidad & $\begin{array}{l}\text { Aspectos } \\
\text { organizacionales }\end{array}$ & $\begin{array}{l}\text { Elementos } \\
\text { personales }\end{array}$ \\
\hline $\begin{array}{l}\text { Colaboración } \\
\text { Intradisciplinar }\end{array}$ & $0.643^{* *}$ & $0.666^{* *}$ & $0.458^{* *}$ \\
\hline ** $<.01$ & & & \\
Fuente: Elaboración propia. & & &
\end{tabular}


En términos de responder a las preguntas de investigación, en relación a la primera interrogante, sobre en qué medida los aspectos institucionales, organizacionales y personales explicarían la colaboración intradisciplinar, tomando en consideración el coeficiente de determinación del modelo de regresión, simbolizado por el valor R2 (tabla 4), que explica el monto de varianza de la variable dependiente explicado por el conjunto de variables independientes, se tiene que este alcanza un valor de 0,514. Ello, situado en el presente análisis, indicaría que el 51,4\% de la variabilidad de la colaboración intradisciplinar estaría siendo explicada por los elementos institucionales en los que se desarrolla la intervención social, en la que participan profesionales y técnicos, los aspectos organizacionales y los elementos personales propios de los interventores.

Por su parte, en relación a cuál de los tres elementos -institucionalidad, aspectos organizacionales y elementos personales - tendría mayor peso explicativo en la colaboración (pregunta de investigación 2), los coeficientes estandarizados del modelo (tabla 4) indican que el mayor peso lo tendría la institucionalidad en la que se sitúa la intervención $(0,354)$. Le seguirían los aspectos organizacionales $(0,301)$ y, finalmente, los personales $(0,187)$. Los resultados del modelo de regresión se presentan en la siguiente tabla:

Tabla 4.

Resultados de la regresión múltiple para explicar cambios en la colaboración intradisciplinar ( $Y$ ) basado en la Institucionalidad (X1), Aspectos Organizacionales (X2) y elementos personales de las y los interventores del Trabajo Social (X3)

\begin{tabular}{|c|c|c|}
\hline $\begin{array}{l}\text { Variable dependiente: Colaboración } \\
\text { Intradisciplinar }\end{array}$ & $\begin{array}{l}\text { Coeficientes } \\
\text { beta }\end{array}$ & $\begin{array}{l}\text { B (coeficiente } \\
\text { estandarizado) }\end{array}$ \\
\hline Institucionalidad & $0,419^{*}$ & 0,354 \\
\hline Aspectos organizacionales & $0,414^{*}$ & 0,301 \\
\hline \multirow[t]{5}{*}{ Elementos personales } & $0,309^{*}$ & 0,187 \\
\hline & $\mathrm{N}$ & 103 \\
\hline & Intercept & $-0,535$ \\
\hline & $\mathrm{R} 2$ & 0,514 \\
\hline & R2 ajustado & 0,500 \\
\hline
\end{tabular}

${ }^{*} \mathrm{p}<.05$

Fuente: Elaboración propia. 
Con los elementos de la tabla se puede construir una ecuación de regresión situada en los datos disponibles, a expresar de la siguiente manera:

Colaboración Intradisciplinar $=-0,535+0,419 *$ Institucionalidad + $0,414^{*}$ Aspectos organizacionales $+0,309 *$ Elementos personales

Dado que en todos los casos relevantes los coeficientes beta son positivos, se aprecian relaciones que en matemáticas se entienden como de "pendiente positiva". Así, la pendiente positiva asociada a la institucionalidad $(0,419)$ indicaría que, manteniendo constantes los aspectos organizacionales y los elementos personales, un aumento de un punto en el acuerdo en relación a cómo la institucionalidad favorecería la colaboración intradisciplinar, aumentaría, en promedio, en 0,419 puntos el acuerdo en relación a experiencias positivas en torno a la colaboración entre profesionales y técnicos del Trabajo Social.

Asimismo, manteniendo lo demás constante, un aumento en un punto en el acuerdo en relación a cómo los aspectos organizacionales favorecerían la colaboración, permitiría aumentar, en promedio, en 0,414 puntos el acuerdo que tendrían los interventores del Trabajo Social en relación a vivencias positivas a la hora de colaborar entre sí en el desarrollo de la intervención.

Finalmente, un aumento en un punto en el acuerdo en relación a cómo los elementos personales favorecerían la colaboración, permitiría aumentar, en promedio, en 0,309 el acuerdo de los interventores en torno a experiencias positivas de colaboración intradisciplinar. Una vez más, manteniendo todo lo demás constante.

\section{Conclusiones}

Este trabajo ha buscado responder en qué medida aspectos institucionales, organizacionales y personales explicarían la colaboración entre profesionales y técnicos del Trabajo Social en Chile en contextos de intervención social. Asimismo, cuál de estos tres elementos tendría un mayor peso explicativo en la colaboración. En este sentido, situado en los datos disponibles en este estudio, se advierte que la institucionalidad, los aspectos organizacionales y los elementos personales explicarían poco más de la mitad de la colaboración intradisciplinar o, dicho de otro modo, los cambios en ella. Cabe rele- 
var en este sentido que, si bien para un estudio en ciencias sociales en general, y con una vertiente exploratoria en particular, este valor podría considerarse alto, la complejidad de los fenómenos sociales rara vez puede ser capturada en su totalidad por modelamientos cuantitativos. Desde ahí emerge como posibilidad explicativa el que se encuentre una sumatoria de elementos no abordados en la construcción del instrumento. Pese a las diversas estrategias seguidas para arribar a un instrumento que posibilitase el abordaje comprehensivo de los diversos elementos de la colaboración, este en ningún caso podría capturar en tiempo real la sumatoria de elementos territoriales, sociales y contextuales en las que intervienen, por ejemplo, personas trabajadoras sociales y técnicas a lo largo de todo Chile. Las realidades son cambiantes, dinámicas y situadas, y, desde este reconocimiento, aproximarse a ellas supone un ejercicio que, a lo menos desde la concepción de este estudio, requiere vincularse epistemológicamente con los instrumentos desde una comprensión de sus bondades y limitaciones, mas no desde una concepción soberbia que los vea como infalibles o en pie de poder capturar realidades en lógicas positivistas de control. Al respecto, reconociendo las bondades del instrumento y los métodos utilizados, este debiese ser permanentemente revisado, ajustado y también complementado con otras técnicas de producción de información.

Por su parte, situado en los datos producidos, es posible concluir que el mayor peso explicativo a la base de la colaboración la tendría la institucionalidad, seguida de los elementos organizacionales. Estos hallazgos dialogan con previas investigaciones en relación con el rol de los aspectos institucionales en materia de colaboración en la intervención (véase, por ejemplo, las contribuciones de Andrade-Guzmán et al., (2014), o de Caplan y Ricciardelli (2016)) o en torno al papel que cumplirían los elementos organizacionales en este ámbito (Calkin, 1988). En este contexto, desde un reconocimiento de que la colaboración se encontraría permeada por estas condiciones estructurales (Galyean et al., 2018; Iachini, Bronstein \& Mellin, 2018), se vuelve un imperativo ético la necesidad de interpelar y demandar que estas provean de las mejores condiciones para el desarrollo de estrategias colaborativas. Ello implicaría, en términos operacionales - por ejemplo, en un nivel institucional-, asegurar un financiamiento digno a los programas de intervención, revisar críticamente aspectos de concur- 
sabilidad vinculados a las intervenciones, los cuales, desde un ethos neoliberal, centrado en la competencia, entran en profunda contradicción con promover prácticas colaborativas entre interventores, que pongan por encima las necesidades de los sujetos. Asimismo, implica revisar críticamente aspectos operativos, como el número de casos con el que han de trabajar los interventores de la disciplina, de manera de contribuir a la generación de condiciones adecuadas para un encuentro intradisciplinar que promueva al máximo posible una colaboración significativa y transformadora.

Por su parte, en el ámbito organizacional, dado el peso que tendrían estos aspectos en la colaboración intradisciplinar, supone necesariamente avanzar hacia el robustecimiento de culturas organizativas que, desde un sentido inmaterial, promuevan, reconozcan y valoren la contribución de los interventores (profesionales y técnicos), pero que también, desde uno material, robustezcan de forma constante las condiciones de operación de los programas, generando y resguardando los tiempos, por ejemplo, para el cuidado de los equipos. Además cautelando, en todos los casos, condiciones dignas de operación y soporte a los programas, como por ejemplo en materia de recursos de implementación, espacios de trabajo, condiciones de contratación, nivel de trabajo administrativo a gestionar, entre otros, de modo de asegurar, en todos los casos, condiciones de operación dignas para quienes se encuentran desarrollando intervenciones en marcos de altísima complejidad, como puede ser la restitución de derechos gravemente vulnerados de niñas, niños y adolescentes, o de personas con diagnósticos asociados a la situación de discapacidad.

A su vez, teniendo los aspectos personales relevancia en la colaboración, es crucial reconocer que, en una lógica agencia-estructura (Cleaver \& De Koning, 2015; Cleaver \& Whaley, 2018), con condiciones de operación, esta última, en ocasiones, no de la mayor solidez (expresada, por ejemplo, en bajos sueldos para los interventores, alta carga de número de casos a atender, entre otros), elementos como el agotamiento de los equipos, la rotación y otros pueden eventualmente poner en tensión la colaboración y los procesos de intervención vinculados con esta. En este sentido, el llamado al reconocimiento y la valoración de la contribución del otro, a la disposición a ceder poder en pos de encontrarse en marcos de horizontalidad, en la presencia 
de condiciones no adecuadas de operación, podría derivar en poner mayoritariamente en las personas integrantes de los equipos, el peso personal y material de llevar adelante procesos de colaboración significativa por la intervención.

Desde esta perspectiva, y desde el posicionamiento crítico desde el que se ha concebido este trabajo, lo anterior, resultaría inaceptable. Reviste entonces la más alta relevancia generar y/o robustecer con un sentido de urgencia, las condiciones institucionales y organizacionales en las que se sitúa la actuación disciplinar de los interventores profesionales y técnicos en Trabajo Social. Lo anterior en el sentido de resignificar y materializar condiciones de operación dignas y del más alto estándar para quienes se dedican a colaborar con otras y otros, en pos de generar mejores condiciones de vida para los sujetos de la intervención social.

\section{Agradecimientos}

Este trabajo ha sido financiado por el Fondo de Fomento a la Investigación, de la Vicerrectoría de Investigación y Postgrado de la Universidad Alberto Hurtado. Ello en el marco del proyecto: DIP2020-1.

\section{Bibliografía}

Aguayo-Cuevas, C., Cornejo-Torres, R. y López-Vásquez, T. (2018). Luces y Sombras del Trabajo Social Chileno. Memoria desde finales de la década del 1950 al 2000: identidad, ética, políticas sociales, formación universitaria y derechos humanos. Buenos Aires, Argentina: Espacio Editorial.

Andrade-Guzmán, C., Martin, M. y Martínez, L. (2014). Protección especial: entre el rol garante del Estado y la prestación de servicios por parte de las organizaciones colaboradoras de SENAME. Santiago de Chile: Centro de Estudios de la Niñez, Corporación OPCIÓN.

Barrow, W., Barrow, R. \& Glockling, T. (2014). Beyond procedures: a case study from educational psychology. In D. Jindal-Snape \& E. F. S. Hannah (Eds.), Exploring the dynamics of ethics (pp. 19-33). Bristol University Press, Policy Press.

Calkin, J. D. (1988). The effects of organizational structure on role behavior. In M. Hardy \& M. Conway (Eds.), Role theory. Pers- 
pectives for health professionals (pp. 133-158). New York: Appleton-Century-Croft

Caplan, M. A., \& Ricciardelli, L. (2016). Institutionalizing neoliberalism: 21st-century capitalism, market sprawl, and social policy in the United States. Poverty and Public Policy, 8(1), 20-38. DOI: https://doi.org/10.1002/pop4.128

Cea, M. (2001). Metodología Cuantitativa: Estrategias y técnicas de investigación social. Madrid, España: Editorial Síntesis.

Cea, M. (2004). Análisis multivariable. Teoría y práctica en la investigación social. España: Editorial Síntesis.

Cleaver, F. (2002). Reinventing Institutions: Bricolage and the Social Embeddedness of Natural Resource Management. The European Journal of Development Research, 14(2), 11-30.

Cleaver, F. \& De Koning, J. (2015). Furthering critical institutionalism. International Journal of the Commons, 9(1), 1-18. DOI: https://doi.org/10.18352/ijc.605

Cleaver, F. \& Whaley, L. (2018). Understanding process, power, and meaning in adaptive governance: A critical institutional reading. Ecology and Society, 23(2). DOI: https://doi. org/10.5751/ES-10212-230249

Galyean, L., Lawson, J., Jones, J., Dreyfus, S. \& Berrick, K. (2018). Child welfare. In A. Iachini, L. Bronstein \& E. Mellin (Eds.), A Guide for Interprofessional Collaboration. United States: Counsil on Social Work Education.

González-Celis, A. y Pérez-Roa, L. (2009). Palabras y cosas para el Trabajo Social: sentidos y significados presentes en las estrategias de intervención utilizadas por los y las Trabajadores Sociales en la Región Metropolitana. Revista Trabajo Social, (76), 65-77.

González, K. (2009). Equipos interdisciplinarios: Nuevas formas de prestar servicios de salud, nuevas oportunidades para el Trabajo Social. Revista Trabajo Social, (76), 79-92.

Guba, E. \& Lincoln, I. (2005). Paradigmatic controversies, contradictions, and emerging confluences. In N. Denzin \& I. Lincoln (Eds.), Handbook of Qualitative Research (pp. 191-215). Thousand Oaks, CA: Sage.

Gujarati, D. y Porter, D. (2010). Econometría. México, México: McGrawHill/ Interamericana Editores, S.A. De C.V. 
Hannah, E. F. S. \& Jindal-Snape, D. (2014). Setting the scene: personal, professional and interprofessional ethics. In D. Jindal-Snape \& E. F. S. Hannah (Eds.), Exploring the dynamics of ethics Book (pp. 3-18). Bristol University Press, Policy Press.

Hodson, A. V. \& Deery, R. (2014). Protecting unborn babies: professional and interprofessional ethical considerations for social work and midwifery. In D. Jindal-Snape \& E. F. S. Hannah (Eds.), Exploring the dynamics of ethics (pp. 151-166). Bristol University Press, Policy Press.

Holland, D. \& Lave, J. (2009). Social Practice Theory and the Historical Production of Persons. Actio: An International Journal of Human Activity Theory, (2), 1-15.

Iachini, A., Bronstein, L. \& Mellin, E. (2018). Structural characteristics. In A. Iachini, L. Bronstein \& E. Mellin (Eds.), A Guide for Interprofessional Collaboration. United States: Counsil on Social Work Education.

Iturrieta, S. (2017). Entre burbujas, sensaciones y realidades de la profesión más masificada en Chile: el Trabajo Social. Cuadernos de Trabajo Social, 1(9), 9-26.

Jacob, J., Boshoff, K., Stanley, R., Stewart, H. \& Wiles, L. (2017). Interprofessional collaboration within teams comprised of health and other professionals: a systematic review of measurement tools and their psychometric properties. Internet Journal of Allied Health Sciences and Practice, 15(2), 1-12.

Katherine, J. \& Georgeson, J. (2013). Personal action potency: early years practitioners participating in interprofessional practice in early years settings. International Journal of Early Years Education, 21(1), 39-55.

Kelly, L. \& Young, J. (2014). Child protection social work in times of uncertainty: dilemmas of personal and professional ethics. In D. Jindal-Snape \& E. F. S. Hannah (Eds.), Exploring the dynamics of ethics (pp. 119-131). Bristol University Press, Policy Press.

López, M. (2010). El camino recorrido. Estudio acerca de cómo hacen y cómo han hecho, trabajadores sociales chilenos. Intervención Comunitaria. Universidad de Chile.

Marradi, A., Archenti, N. y Piavoni, J. (2010). Metodología de las ciencias sociales. Buenos Aires, Argentina: Cengage learning Argentina. 
Morgan, S., Pullon, S. \& Mckinlay, E. (2015). Observation of interprofessional collaborative practice in primary care teams: An integrative literature review. International Journal of Nursing Studies, 52(7), 1217-1230. DOI: https://doi.org/10.1016/j.ijnurstu.2015.03.008

Muñoz-Arce, G. (2014). Aportes conceptuales y empíricos para pensar la intervención social interdisciplinar en Chile. Intervención, (3), 19-29.

Muñoz-Arce, G. (2016). Critical social work and the promotion of citizenship in Chile. International Social Work, 1-13. DOI: https://doi.org/10.1177/0020872816664965

Muñoz-Arce, G. (2018). The neoliberal turn in Chilean social work: frontline struggles against individualism and fragmentation. European Journal of Social Work, O(0), 1-12. DOI: https:// doi.org/10.1080/13691457.2018.1529657

Muñoz-Arce, G. y Madrigal-Calderón, J. (2018). Trabajo interprofesional en Chile: neoliberalismo y la producción de subjetividades profesionales críticas. Intervención, 8(2), 4-22.

Orchard, C. A. (2010). Persistent isolationist or collaborator? The nurse's role in interprofessional collaborative practice. Journal of Nursing Management, 18, 248-257. DOI: https://doi. org/10.1111/j.1365-2834.2010.01072.x

Pérez, G. (1994). Investigación cualitativa. Retos e interrogantes. Madrid, España: La Muralla.

Ross, T. (2009). Child Welfare. The challenges of collaborations. Washington, United States: The Urban Institute Press.

Sharland, E. (2012). All Together Now? Building Disciplinary and InterDisciplinary Research Capacity in Social Work and Social Care. The British Journal of Social Work, 42(2), 208-226. DOI: https://doi.org/10.1093/bjsw/bcr06l

Smith, D. C. (2015). Midwife - Physician Collaboration: A Conceptual Framework for Interprofessional Collaborative Practice. Journal of Midwifery \& Women's Health, 60(2), 128-139. DOI: https://doi.org/10.1111/jmwh.12204

Stake, R. (1999). Investigación con estudio de casos. Ediciones Morata.

Vidal, P. (2015). La educación del trabajo social en Chile: hacia un siglo de historia. Quaderni Del Csal, 3, 177-192. 
West, A., Miller, S. \& Leitch, J. (2016). Professional Socialization and Attitudes Towards Interprofessional Collaboration Among Graduate Social Work and Health Professions Students. Advances in Social Work, 17(2), 134-150. DOI: https://doi. org/10.18060/19809 